Introduction Endothelial outgrowth cells (EOC) isolated from the circulation can be expanded in culture and have major potential as a therapy for vascular regeneration, but investigation of their role in vascular repair has been limited by the lack of a safe model of arterial injury in man. We developed a GMP-compliant culture process for EOC production and characterised the functional response to vascular injury in patients undergoing transradial cardiac catheterization.

Methods Patients with stable angina $(\mathrm{n}=50,63.8 \pm 10.8$ years) undergoing transradial cardiac catheterization had an assessment of flow-mediated dilatation (FMD) in both radial arteries prior to catheterisation and at 24 h, 1, 4 and 12 weeks. Peripheral blood was obtained for the isolation and quantification of EOC, and for the development of a GMP-complaint protocol.

Results FMD was attenuated in the catheterised artery compared to the non-catheterised artery at $24 \mathrm{~h}(4.3 \pm 3.4$ vs. $10.7 \pm 5.6, \mathrm{P}<0.05)$ and at one week $(3.1 \pm 4.1$ vs. 7.7 $\pm 5.6, \mathrm{P}<0.05)$, but had recovered by 12 weeks. EOCs were obtained in $66.67 \%$ of patients (14/21) and using a GMP-compliant protocol we were able to produce $32.1 \pm$ $11.2 \times 10^{6}$ cells over four weeks.

Conclusions Transradial catheterization is associated with endothelial denudation and radial artery vasomotor dysfunction, and represents an accessible and reproducible model of vascular injury in man. The development of an efficient GMP-compliant culture protocol for the manufacture of EOC will permit first-in-man clinical trials to assess whether EOCs are able to home to and incorporate at sites of vascular injury in man.

\section{TIMING OF SURGICAL AND PERCUTANEOUS REVASCULARISATION FOR LEFT MAIN STEM CORONARY ARTERY DISEASE IN THE WEST OF SCOTLAND}

Alice M Jackson*, Peter McCartney, Richard Good. Golden Jubilee National Hospital; *Presenting Author

\subsection{6/heartjnl-2016-309890.33}

Introduction Significant ( $>50 \%$ ) left main stem stenosis (LMS) is found in $4-6 \%$ of patients undergoing diagnostic angiography. Unprotected LMS lesions are associated with a poor prognosis and the mortality for non-revascularised disease may be as high as $37 \%$ at 3 years. In order to reduce morbidity and mortality associated with delayed treatment, European guidelines recommend that all patients with LMS disease undergo revascularisation within 2 weeks.

Aims We audited all patients in our high-volume tertiary centre that underwent revascularisation by coronary artery bypass grafting (CABG) or percutaneous coronary intervention (PCI) for significant LMS stenosis during a 6-month period. We sought to investigate timing between coronary angiography and revascularisation for stable and unstable presentations and compare our findings to the international guidelines.

Methods Patients with significant LMS disease observed during invasive coronary angiography (Feb-Aug 2014) were retrospectively identified using the coronary intervention patient-database at the West of Scotland Regional Heart and Lung Centre (Golden Jubilee National Hospital). Data were collected from angiography reports and electronic patient records. Patients who did not undergo revascularisation or who had primary PCI were excluded from the analyses. Patients were considered unstable if the indication for angiography was acute coronary syndrome, including STelevation myocardial infarction without primary PCI.

\begin{tabular}{|c|c|c|c|}
\hline & DUI(n-ISS) & CABG (6नIDU) & PCI (n-62) \\
\hline Mule sen an $(x)$ & $125(76)$ & $83(81)$ & $42(68)$ \\
\hline \multicolumn{4}{|l|}{$\operatorname{Ag}=n(N)$} \\
\hline $20-39$ years & $1(1)$ & $1(1)$ & 0 \\
\hline 40-59 yours & $33(20)$ & $27(26)$ & $6(10)$ \\
\hline $60-79$ years & $102(62)$ & 63 (58) & $39(63)$ \\
\hline abe yours & $29(18)$ & $12(12)$ & $17(27)$ \\
\hline \multicolumn{4}{|l|}{ Cenortidities $1(\boldsymbol{N})$} \\
\hline Dubetes & $\mathbf{4 ( 2 7 )}$ & $24(23)$ & $20(32)$ \\
\hline Previoer enyocardial infarction & 58 (35) & $31(30)$ & $27(44)$ \\
\hline Previloses atrede & $18(11)$ & 11 (11) & $7(11)$ \\
\hline Neripheral vasedur disedere & $22(13)$ & $9(9)$ & $13[21]$ \\
\hline \multicolumn{4}{|l|}{ Anclogram indicatien $n$ (W) } \\
\hline Suble & $93(56)$ & $68(66)$ & $25(40)$ \\
\hline Unstable & $n(44)$ & $35(34)$ & $37(60)$ \\
\hline \multicolumn{4}{|l|}{$\begin{array}{l}\text { Time te revaculariatiog on (W) } \\
\text { s14dwys }\end{array}$} \\
\hline all & 63 (38) & $20(21)$ & $43(69)$ \\
\hline Stable & $16\left[25^{n}\right]$ & $5\left(25^{\star}\right)$ & $11\left(26^{n}\right)$ \\
\hline Unatable & $47\left(75^{n}\right)$ & $15\left(75^{n}\right)$ & $32\left(74^{n}\right)$ \\
\hline \multicolumn{4}{|l|}{$>14 d a y s$} \\
\hline All & $102(62)$ & $83(81)$ & 19 [31] \\
\hline Suble & $n\left[75^{n}\right]$ & $63\left(76^{\circ}\right)$ & $14\left(74^{n}\right)$ \\
\hline Unatable & $25\left(25^{n}\right)$ & $20\left(24^{\star}\right)$ & $5\left(266^{*}\right)$ \\
\hline
\end{tabular}

Abstract 33 Table 1 Baseline character

Results 207 patients were found to have significant LMS disease during the audit period, 42 were excluded (not revascularised or primary PCI). A total of 165 patients underwent non-emergency revascularisation. 103/165 (62\%) patients were revascularised by CABG. A larger proportion of patients in the PCI group were female, older, had additional comorbidities and were unstable at presentation compared with the CABG group (Table 1). 102/165 (62\%) patients waited >14 days for revascularisation. Figure 1 shows the distribution of timing of revascularisation by CABG and PCI, highlighting the stability of patients within each group. 16/93 (17\%) stable patients and $47 / 72(65 \%)$ unstable patients were revascularised within 14 days, with the majority undergoing PCI (43/64, $68 \%)$. The main reason for delay $>14$ days for CABG was the wait for outpatient surgery (Figure 2). In the PCI group waiting >14 days, 5/19 (26\%) patients were referred back for PCI after being deemed unsuitable for surgery (Figure 3).

Conclusion Huge service developments have been made in recent years to deliver timely revascularisation for patients, particularly those with unstable coronary disease. Despite this, we have found that patients with the highest risk coronary anatomy are experiencing significant delays to revascularisation, particularly by CABG. Further efforts to streamline the

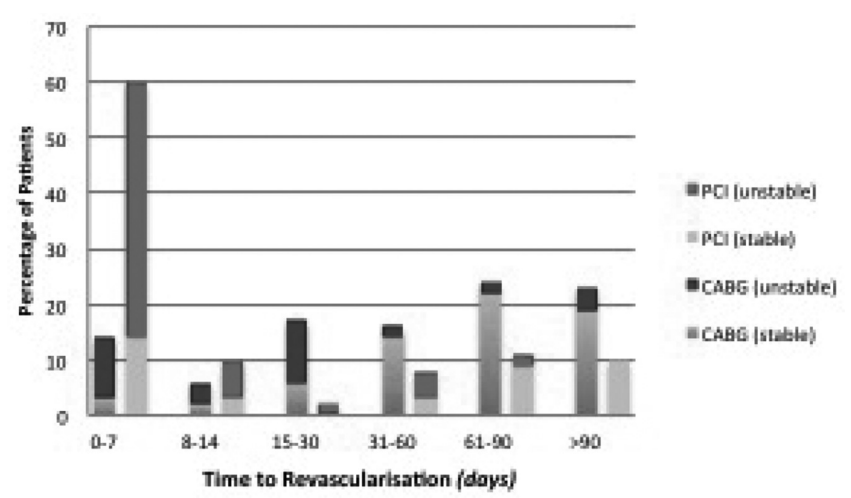

Abstract 33 Figure 1 Time to revascularisation stratified by patient stability 


\section{CABG (n=83)}

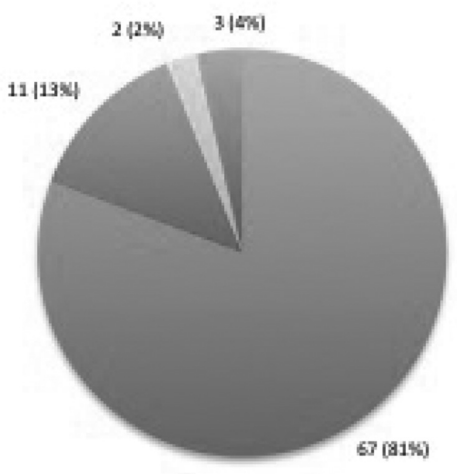

PCI (n-19)

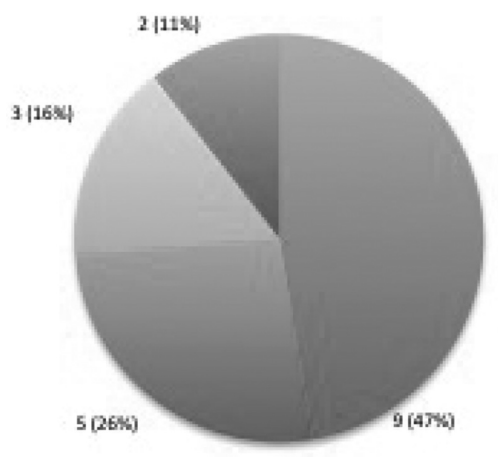

Abstract 33 Figure 2 Reasons for delay $>14$ days for CABG and $\mathrm{PCI}$

pathway to both surgical and percutaneous revascularisation for this patient group are needed.

\section{DO CENTRES THAT USUALLY PERFORM PERCUTANEOUS CORONARY INTERVENTION TRANS-RADIALLY HAVE INFERIOR OUTCOMES WHEN OPERATING TRANS- FEMORALLY?}

${ }^{1}$ William Hulme* ${ }^{1}$ Matthew Sperrin, ${ }^{1}$ Evan Kontopantelis, ${ }^{2}$ Peter Ludman, ${ }^{3}$ Mark de Belder, ${ }^{4}$ James Nolan, ${ }^{4}$ Mamas A Mamas. ${ }^{1}$ Farr Institute; ${ }^{2}$ Queen Elizabeth Hospital; ${ }^{3}$ James Cook University Hospital; ${ }^{4}$ Keele Cardiovascular Research Group; * Presenting Author

\subsection{6/heartjnl-2016-309890.34}

Background Over the last decade trans-radial access (TRA) has become more common than trans-femoral access (TFA) for Percutaneous Coronary Intervention (PCI) in the UK. Despite studies highlighting the benefits of this transition, there are concerns that the resulting drop in TFA activity has led to operators and centres losing TFA proficiency, compromising the safety and efficacy of procedures where TFA is necessary. Aims To evaluate the impact of each centre's recent experience of the TFA approach on procedural outcomes in TFA-only procedures.

Methods This retrospective cohort study used procedures recorded in the British Cardiovascular Intervention Society (BCIS) PCI audit from 2007 to 2013 in England and Wales. Centres were split into one of three groups depending on the proportion of total procedures undertaken via TFA in 2013, with patient and procedural characteristics for TFA-only procedures observed within these groups over time. By considering each centre's access site choices in the 12 months prior to procedure date, simply-derived measures were used to capture the 'recent TFA experience' of the operating centre for each procedure. The association of these measures on 30-day mortality, after risk-adjustment, were then studied using multiple logistic regression.

Results A total of 235,474 procedures were available for analysis. Unadjusted TFA-only mortality in centres who were early-adopters of TRA increased more rapidly than for centres who maintained high TFA activity, and this was driven by higher baseline risk. After case-mix adjustment, recent TFA experience was found to have no effect on 30-day mortality $(\mathrm{OR}=0.99$ per 0.1 increase in recent TFA proportion; $\mathrm{CI}=0.96$ to $1.01 ; \mathrm{p}=0.220$ ), with similar results when restricting to procedures with low clinical-complexity $(\mathrm{OR}=0.98$ per 0.1 increase in recent femoral proportion; $\mathrm{CI}=0.95$ to $1.01 ; \mathrm{p}=0.245)$.

Conclusions Poorer TFA outcomes for predominantly high TRA centres are driven by the propensity of these centres to utilise TFA in the highest risk patients. Once differences in case mix are adjusted for, TFA outcomes are similar between high and low radial proportion centres, with no evidence to suggest that increasing unfamiliarity with the TFA technique is detrimental. The outcome gains achieved by the national adoption of TRA is not attenuated by a loss of TFA proficiency, and centres should be encouraged to continue to adopt TRA as the default access site for PCI wherever possible.

\section{THROMBUS ASPIRATION DOES NOT REDUCE MORTALITY IN STEMI PATIENTS: A META-ANALYSIS OF 20,192 PATIENTS, WITH IMPLICATIONS FOR FUTURE TRIAL DESIGN}

Yousif Ahmad*, Sayan Sen, Sukhjinder Nijjer, Daniel Keene, Chris Cook, Ricardo Petraco Matthew Shun-Shin, Graham Cole, Rasha Al-Lamee, Iqbal Malik, Christopher Baker, Ghada Mikhail, Rodney Foale, Jamil Mayet, Justin Davies, Darrel Francis. Imperial College London; *Presenting Author

\subsection{6/heartjnl-2016-309890.35}

Background Thrombus aspiration is a mechanistically logical adjunct in primary angioplasty for acute myocardial infarction. Individual randomised controlled trials (RCTs) have not shown a consensus of mortality reduction and there are concerns about stroke. We perform a meta-analysis of all available RCT data on thrombus aspiration, including the large, recently published TOTAL trial.

Method and results A meta-analysis of RCTs of thrombus aspiration, including the recent TOTAL data was performed. At 30 days (11 trials; 20,192 patients) there was a marginally non-significant reduction in all cause-mortality with thrombus aspiration (OR $0.844,95 \%$ CI $0.710-1.003, p=0.05$ ). The longer-term follow-up data (13 trials; 20,142 patients) was similarly non-significant (OR $0.89,95 \%$ CI $0.78-1.01, \mathrm{p}=$ 0.08). At both 30 days and longer-term follow-up, there was a statistically significant increase in stroke with thrombus aspiration $(\mathrm{OR} 1.56,95 \% \mathrm{CI} 1.05$ to $2.32, \mathrm{p}=0.03$, and $\mathrm{OR}$ $1.94,95 \%$ CI 1.24 to $3.04, \mathrm{p}=0.04$ respectively).

Conclusions The point estimates in the meta-analyses suggest that thrombus aspiration may prevent four deaths per thousand at the cost of two strokes per thousand. Although this 